\title{
Correction to: Curcumin in Advancing Treatment for Gynecological Cancers with Developed Drug- and Radiotherapy- Associated Resistance
}

Amir Abbas Momtazi-Borojeni, Jafar Mosafer, Banafsheh Nikfar, Mahnaz Ekhlasi-Hundrieser, Shahla Chaichian, Abolfazl Mehdizadehkashi, and Atefeh Vaezi

\section{Correction to:}

Chapter "Curcumin in Advancing Treatment for Gynecological Cancers with Developed Drugand Radiotherapy-Associated Resistance" in:

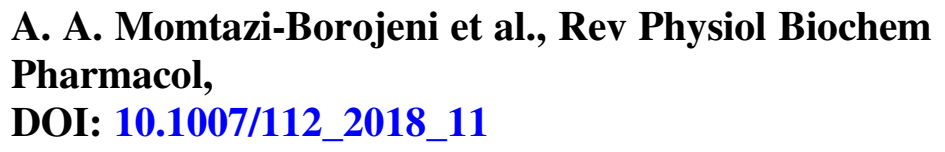

The affiliation of the 6th author Dr. Abolfazl Mehdizadehkashi was incorrect. It has been corrected to Endometriosis Research Center, Iran University of Medical Sciences, Tehran, Iran. 